\title{
EXISTENCE OF POSITIVE SOLUTIONS FOR SINGULAR ORDINARY DIFFERENTIAL EQUATIONS WITH NONLINEAR BOUNDARY CONDITIONS
}

\author{
L. E. BOBISUD AND DONAL O'REGAN \\ (Communicated by Hal L. Smith)
}

\begin{abstract}
We prove the existence of nonnegative solutions of the problem

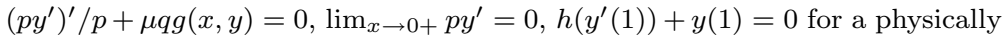
motivated class of nonlinearity $h$. The results, which are established using a "forbidden value" argument, are new even in the case of linear $h$.
\end{abstract}

\section{INTRODUCTION}

Consider a thin rod with internal heat generation or lateral surface heating occupying the interval $0 \leq x \leq 1$; a mathematical model for the steady-state absolute temperature $y$ in the rod is

$$
y^{\prime \prime}(x)+\mu q(x) g(x, y(x))=0
$$

for suitable $q$ and $g$. Here $\mu q(x)$ may be interpreted as the reciprocal of the local heat conductivity and $g$ as the law governing heat generation as a function of position and temperature. We assume that the boundary at $x=0$ is insulated, so $y^{\prime}(0)=0$. As prototype boundary condition at $x=1$ we consider Stefan's and Newton's laws: $-k y^{\prime}(1)=y(1)^{\alpha}-T^{\alpha}$, where $k$ is a positive constant, $T$ is the ambient temperature, and $\alpha=4$ or $\alpha=1$, respectively. We prefer to rewrite the boundary condition in the form

$$
h\left(y^{\prime}(1)\right)+y(1)=0,
$$

where

$$
h(z)=-\left(T^{\alpha}-k z\right)^{1 / \alpha} \quad \text { for } \quad z \leq T^{\alpha} / k .
$$

We suppose for convenience that this $h$ is artificially extended to $z>T^{\alpha} / k$ as a positive increasing function. Abstracting from these examples, it is natural to assume that

H1. $h:(-\infty, \infty) \rightarrow(-\infty, \infty)$ is a continuous, nondecreasing function, and there exists a unique $\beta \geq 0$ such that $h(\beta)=0$.

Received by the editors January 15, 1995.

1991 Mathematics Subject Classification. Primary 34B15.

Key words and phrases. Boundary value problems, nonlinear boundary conditions, nonlinear alternative. 
In the theorems to follow it will be shown that in fact a solution $y$ satisfies $y^{\prime}(1) \leq \beta$. Our concern is to determine a class of functions $h$ satisfying $\mathrm{H} 1$ and for which a solution to our problem exists for some range of $\mu>0$.

We generalize the problem above to include symmetric solutions of symmetric heat conduction problems in higher dimensions by examining the problem

$$
\begin{gathered}
\frac{1}{p(x)}\left(p(x) y^{\prime}(x)\right)^{\prime}+\mu q(x) g(x, y(x))=0, \\
\lim _{x \rightarrow 0+} p(x) y^{\prime}(x)=0, \quad h\left(y^{\prime}(1)\right)+y(1)=0 ;
\end{gathered}
$$

a different boundary condition at $x=0$ could be considered, but that of (2) stems naturally from elliptic partial differential equations. For such equations in $R^{n}$ we have that $p(x)=x^{n-1}$ for solutions depending only on the radial variable $x$; we therefore do not assume that $(1 / p) \in L_{1}(0,1)$, since to do so would eliminate from consideration elliptic problems in two and more spatial dimensions.

Even for the case of a linear boundary condition at $x=1$, the theorems of this paper are stronger than similar results in the literature because we use a "forbidden value" type argument [1]. Thus a major hypothesis of each of our theorems requires the supremum of a certain quantity to be sufficiently large, a weaker condition than the usual requirement that the limit of this quantity be large. Essentially, the supremum determines a forbidden value of the norm of solutions, i.e., a value that the norm never takes on. This suffices for the nonlinear alternative, which requires only the existence of a suitable open set with no fixed points (= solutions) on its boundary. In the usual application, all solutions are shown to lie in this open set, whereas we show only the weaker result that no solution lies on the boundary. Thus our technique is able to handle existence for problems wherein the set of norms of all solutions is unbounded.

Erbe and Wang [2] establish existence of solutions of $u^{\prime \prime}+a(t) F(u)=0$ on $[0,1]$ under general linear homogeneous boundary conditions at 0 and at 1 ; they assume that one of

$$
\lim _{u \rightarrow 0} \frac{F(u)}{u}, \quad \lim _{u \rightarrow \infty} \frac{F(u)}{u}
$$

is infinite and the other is zero. Our Theorem 1 below yields their result (for our boundary conditions); indeed, for a linear boundary condition at $x=1$ we require only that $\sup _{z \in(0, \infty)}(z / f(z))$ exceed a certain constant, where $f$ is a nondecreasing function satisfying $F(u) \leq f(u)$.

\section{EXISTENCE THEOREMS}

In view of the connection with symmetric problems for elliptic partial differential equations, we allow the coefficient $p$ to vanish at zero. We also allow $q$ to be singular at $x=0$ and $x=1$. Specifically, we require

H2. $p$ is continuous and strictly positive on $(0,1]$.

H3. $q \in \mathrm{C}(0,1)$ satisfies $q>0$ on $(0,1)$, and

$$
\int_{0}^{1} p(s) q(s) d s<\infty, \quad \int_{0}^{1} \frac{1}{p(t)} \int_{0}^{t} p(s) q(s) d s d t<\infty .
$$

H4. $g:[0,1] \times[0, \infty) \rightarrow(-\infty, \infty)$ is continuous, $g(t, 0) \geq 0$ for $t \in(0,1)$, and there exists a continuous nondecreasing function $f:[0, \infty) \rightarrow[0, \infty)$ such that $f(y)>0$ for $y>0$ and $g(t, y) \leq f(y)$ holds on $(0,1) \times(0, \infty)$. 
Theorem 1. Assume H1-H4 hold. Set

$$
r_{0}=\max \left\{\frac{1}{p(1)} \int_{0}^{1} p(x) q(x) d x, \int_{0}^{1} \frac{1}{p(x)} \int_{0}^{x} p(t) q(t) d t d x\right\}
$$

and let $\mu_{0}$ satisfy

$$
\sup _{z \in(0, \infty)} \frac{z}{\mu_{0} r_{0} f(z)-h\left(-\mu_{0} r_{0} f(z)\right)}>1 .
$$

Then (1)-(2) has a nonnegative solution if $0 \leq \mu \leq \mu_{0}$.

We remark that the supremum in the condition above is allowed to be infinite.

Proof. As usual in fixed-point arguments of this kind, we consider the oneparameter family of problems

$$
\begin{gathered}
\frac{1}{p}\left(p y^{\prime}\right)^{\prime}+\lambda \mu q g^{*}(x, y)=0, \\
\lim _{x \rightarrow 0+} p(x) y^{\prime}(x)=0, \quad \lambda h\left(y^{\prime}(1)\right)+y(1)=0
\end{gathered}
$$

for $\lambda \in(0,1]$, where we define $g^{*}$ on $[0,1] \times(-\infty, \infty)$ by

$$
g^{*}(x, y)= \begin{cases}g(x, 0)-y, & y<0 \\ g(x, y), & y \geq 0\end{cases}
$$

We first show that any solution of $(3)_{\lambda}$ is nonnegative and hence a solution of $(3)_{\lambda}$ with $g^{*}$ replaced by $g$. If for some $\lambda>0$ the solution $y$ had a negative minimum at $x_{0} \in(0,1)$, then from $(3)_{\lambda}$ we would have $0 \geq g^{*}\left(x_{0}, y\left(x_{0}\right)\right)=g\left(x_{0}, 0\right)-y\left(x_{0}\right)>0$, a contradiction. If a negative minimum occurs at $x_{0}=0$, then the differential equation shows that $\left(p y^{\prime}\right)^{\prime}<0$ as far to the right of zero as $y$ remains negative, whence $y^{\prime}<0$ there, a contradiction. If a negative minimum occurs at $x_{0}=1$, then $y^{\prime}(0) \leq 0$, so from the boundary condition at 1 and $\mathrm{H} 1$ we have that $0>y(1)=$ $-\lambda h\left(y^{\prime}(1)\right) \geq 0$, again a contradiction. Consequently $y \geq 0$ on $[0,1]$. From this we get that $h\left(y^{\prime}(1)\right) \leq 0$, and so $y^{\prime}(1) \leq \beta$.

For notational convenience set

$$
y_{1}=\max \left\{\sup _{[0,1]} y, \sup _{[0,1]} p\left|y^{\prime}\right|\right\} .
$$

Now for $x \in[0,1]$ we have that

$$
\begin{aligned}
y(x)= & \lambda \mu \int_{x}^{1} \frac{1}{p(t)} \int_{0}^{t} p(s) q(s) g^{*}(s, y(s)) d s d t \\
& -\lambda h\left(-\frac{\lambda \mu}{p(1)} \int_{0}^{1} p(t) q(t) g^{*}(t, y(t)) d t\right)
\end{aligned}
$$

and therefore

$$
\begin{aligned}
y(x) \leq & \lambda \mu f\left(y_{1}\right) \int_{0}^{1} \frac{1}{p(t)} \int_{0}^{t} p(s) q(s) d s d t \\
& -\lambda h\left(-\lambda \mu f\left(y_{1}\right) \frac{1}{p(1)} \int_{0}^{1} p(s) q(s) d s\right) \\
\leq & \mu r_{0} f\left(y_{1}\right)-h\left(-\mu r_{0} f\left(y_{1}\right)\right),
\end{aligned}
$$


or

$$
\frac{y(x)}{\mu r_{0} f\left(y_{1}\right)-h\left(-\mu r_{0} f\left(y_{1}\right)\right)} \leq 1
$$

Also,

So

$$
p(x) y^{\prime}(x)=-\lambda \mu \int_{0}^{x} p(t) q(t) g^{*}(t, y(t)) d t
$$

$$
\max _{[0,1]}\left|p y^{\prime}\right| \leq \mu r_{0} f\left(y_{1}\right)
$$

From these two inequalities we get that

$$
\frac{y_{1}}{\mu r_{0} f\left(y_{1}\right)-h\left(-\mu r_{0} f\left(y_{1}\right)\right)} \leq 1 .
$$

Now fix $\mu \leq \mu_{0}$ and let $M_{0}$ satisfy

$$
\frac{M_{0}}{\mu r_{0} f\left(M_{0}\right)-h\left(-\mu r_{0} f\left(M_{0}\right)\right)}>1
$$

Let $B$ be the Banach space $\left\{y \in \mathrm{C}[0,1] \cap \mathrm{C}^{1}(0,1]: p y^{\prime} \in \mathrm{C}[0,1]\right\}$ equipped with the norm

$$
|y|_{1}=\max \left\{\max _{[0,1]}|y|, \max _{[0,1]} p\left|y^{\prime}\right|\right\} \text {. }
$$

Set

$$
\mathrm{C}_{0}^{1}[0,1]=\left\{u \in \mathrm{C}[0,1] \cap \mathrm{C}^{1}(0,1]: p u^{\prime} \in C[0,1] \text { and } \lim _{x \rightarrow 0+} p(x) u^{\prime}(x)=0\right\} \subset B
$$

and define the mapping $N$ on $\mathrm{C}_{0}^{1}[0,1]$ by

$$
(N y)(x)=-h\left(y^{\prime}(1)\right)+\mu \int_{x}^{1} \frac{1}{p(s)} \int_{0}^{s} p(t) q(t) g^{*}(t, y(t)) d t d s .
$$

By the second inequality of $\mathrm{H} 3, N y \in \mathrm{C}[0,1]$ and

$$
(N y)^{\prime}(x)=\frac{-\mu}{p(x)} \int_{0}^{x} p(t) q(t) g^{*}(t, y(t)) d t \in \mathrm{C}(0,1]
$$

Thus $N: \mathrm{C}_{0}^{1}[0,1] \rightarrow \mathrm{C}_{0}^{1}[0,1]$. Continuity of $g^{*}$ and $\mathrm{H} 3$ show that $N$ is continuous as a map in $\mathrm{C}_{0}^{1}[0,1]$. Moreover, the Ascoli-Arzela theorem shows that $N$ maps bounded sets into compact sets. In addition, the fixed points of $\lambda N$ are precisely the solutions of $(3)_{\lambda}$.

Let

$$
U=\left\{u \in \mathrm{C}_{0}^{1}[0,1]:|u|_{1}<M_{0}\right\},
$$

an open subset of the convex subset $\mathrm{C}_{0}^{1}[0,1]$ of $B$. Should there exist $\lambda \in(0,1)$ and $y \in \partial U$ with $y=\lambda N y$, then $y$ is a solution of $(3)_{\lambda}$ satisfying $|y|_{1}=M_{0}$ and hence from (4) we have that

$$
\frac{M_{0}}{\mu r_{0} f\left(M_{0}\right)-h\left(-\mu r_{0} f\left(M_{0}\right)\right)} \leq 1
$$


contradicting the choice of $M_{0}$ in (5). Since $0 \in U$, the nonlinear alternative [3,4] guarantees that $N$ has a fixed point.

Remarks. (1) Note that we do not claim that all solutions satisfy $|y|_{1} \leq M_{0}$, but only that the weaker condition $|y|_{1} \neq M_{0}$ is satisfied by any solution $y$ of $(3)_{\lambda}$ for $\lambda \in[0,1]$.

(2) Minimal adjustments to the argument are required to handle the more general equation

$$
\frac{1}{p}\left(p y^{\prime}\right)^{\prime}+\mu f\left(x, y, p y^{\prime}\right)=0
$$

subject to the same boundary conditions (2).

(3) This existence theorem, as well as the next, has the following physical interpretation in the case of heat conduction: for suitable functional forms for $p, q$, $g$, and $h$, a steady-state solution exists provided the heat conductivity $1 /(\mu q)$ is sufficiently large. This is physically reasonable, for if heat conduction is large, one can expect internally generated heat to be conducted to, and dissipated through, the boundary at $x=1$ as fast as it is generated. In a similar vein, the hypothesis H3 may be viewed as requiring that local regions of poor conductivity not be too extensive. The final condition of the theorem requires also that the heat generation $g$ not be too large.

If a steady-state temperature distribution does not exist, one would expect solutions of the corresponding time-dependent heat equation to "blow up" in some fashion as time increases.

In the previous theorem we produced a value $M_{0}$ such that $|y|_{1} \neq M_{0}$ for any solution $y$ of $(3)_{\lambda}, \lambda \in(0,1]$. In the proof of the following result we are able to find only a forbidden value for $|y|_{0}$; but this suffices, as will be seen.

In this theorem we set $F(z)=\int_{0}^{z} f(u) d s$.

Theorem 2. Let $\mathrm{H} 1, \mathrm{H} 2, \mathrm{H} 3$, and the following hold:

H5. $g \in \mathrm{C}((0,1) \times(0, \infty))$, and $0 \leq g(x, y) \leq f(y)$ on $(0,1) \times(0, \infty)$, where $f$ is continuous and nondecreasing on $(0, \infty)$.

H6. There exists a continuous nondecreasing function $Q(t)$ satisfying

$$
p(t)^{2} q(t) \leq Q(t) \text { on }(0,1) \quad \text { and } \quad \int_{0}^{1} \frac{\sqrt{Q(x)}}{p(x)} d x<\infty .
$$

H7. With

$$
P \equiv \frac{1}{p(1)} \int_{0}^{1} p(t) q(t) d t
$$

$\mu_{0}$ satisfies

$$
\sup _{0<s<\infty} \int_{-h\left(-\mu_{0} P f(s)\right)}^{s} \frac{d z}{\sqrt{F(s)-F(z)}}>\sqrt{2 \mu_{0}} \int_{0}^{1} \frac{\sqrt{Q(x)}}{p(x)} d x .
$$

Then (1)-(2) has at least one nonnegative solution for $\mu \in\left(0, \mu_{0}\right]$.

Proof. Note that the left hand side of (6) increases as $\mu_{0}$ decreases, so if this inequality holds for some $\mu_{0}$, it also holds for any smaller value. $P$ exists by $\mathrm{H} 3$. 
We consider the family of problems

$$
\begin{gathered}
\frac{1}{p}\left(p y^{\prime}\right)^{\prime}+\lambda \mu q g(x, y)=0, \\
\lim _{x \rightarrow 0+} p(x) y^{\prime}(x)=0, \quad \lambda h\left(y^{\prime}(1)\right)+y(1)=0
\end{gathered}
$$

for $\lambda \in(0,1]$. For some $\lambda$ and $\mu$ let $y$ be any nonnegative solution of this problem; then $\left(p y^{\prime}\right)^{\prime} \leq 0$, so $y$ is nonincreasing. In particular, we have that $y^{\prime}(1) \leq 0 \leq \beta$, as promised in the introduction. Multiplying $(7)_{\lambda}$ by $p^{2} y^{\prime}$ and integrating over $(0, x) \subset(0,1)$ leads us to

$$
p(x)^{2} y^{\prime}(x)^{2} \leq-2 \lambda \mu \int_{0}^{x} p(t)^{2} q(t) f(y(t)) y^{\prime}(t) d t \leq 2 \mu Q(x) \int_{y(x)}^{y(0)} f(u) d u
$$

since $y^{\prime} \leq 0$ and $\lambda \leq 1$. Separation of variables and a further integration over $[0,1]$ yields

$$
\int_{y(1)}^{y(0)} \frac{d z}{\sqrt{F(y(0))-F(z)}} \leq \sqrt{2 \mu} \int_{0}^{1} \frac{\sqrt{Q(x)}}{p(x)} d x .
$$

Now $y$ must satisfy the integral equation

$$
\begin{aligned}
y(x) & =\lambda \mu \int_{x}^{1} \frac{1}{p(t)} \int_{0}^{t} p(s) q(s) g(s, y(s)) d s d t \\
& -\lambda h\left(-\frac{\lambda \mu}{p(1)} \int_{0}^{1} p(t) q(t) g(t, y(t)) d t\right),
\end{aligned}
$$

from which it follows that

$$
y(1)=-\lambda h\left(-\frac{\lambda \mu}{p(1)} \int_{0}^{1} p(t) q(t) g(t, y(t)) d t\right) \leq-h(-\mu P f(y(0)))
$$

since $y(1) \geq 0$. Thus from (10) we see that any solution $y$ must satisfy

$$
\int_{-h(-\mu P f(y(0)))}^{y(0)} \frac{d z}{\sqrt{F(y(0))-F(z)}} \leq \sqrt{2 \mu} \int_{0}^{1} \frac{\sqrt{Q(x)}}{p(x)} d x .
$$

Let $\mu_{0}$ satisfy (6); then $\mu \leq \mu_{0}$ also satisfies (6) and there must exist a value $M_{0} \in(0, \infty)$ such that

$$
\int_{-h\left(-\mu P f\left(M_{0}\right)\right)}^{M_{0}} \frac{d z}{\sqrt{F\left(M_{0}\right)-F(z)}}>\sqrt{2 \mu} \int_{0}^{1} \frac{\sqrt{Q(x)}}{p(x)} d x .
$$

In view of (11), $M_{0}$ is a forbidden value for $y(0)=|y|_{0}$. From (9) we see that any solution $y$ of $(7)_{\lambda}-(8)_{\lambda}$ that satisfies $0 \leq y(x) \leq M_{0}$ on $[0,1]$ also satisfies

$$
p(x)^{2}\left|y^{\prime}(x)\right|^{2} \leq 2 \mu Q(1) \int_{y(1)}^{y(0)} f(u) d u \leq 2 \mu Q(1) \int_{0}^{M_{0}} f(u) d u \equiv M_{1}^{2} .
$$


Let $B$ be the Banach space introduced in the proof of Theorem 1 but with the equivalent norm

$$
\|u\|_{1}=\max \left(\max _{x \in[0,1]}|u(x)| / M_{0}, \max _{x \in[0,1]} p(x)\left|u^{\prime}(x)\right| / M_{1}\right) .
$$

It follows that no nonnegative solution of $(7)_{\lambda}-(8)_{\lambda}$ for $\lambda \in(0,1)$ lies on the boundary of

$$
U=\left\{u \in B:\|u\|_{1}<1\right\} .
$$

There is no loss of generality in supposing $g(x, y)$ extended to negative values of $y$ as a nonnegative, continuous function. Let $y$ be any solution of $(7)_{\lambda}-(8)_{\lambda}$ with $g$ so extended. Then $\left(p y^{\prime}\right)^{\prime} \leq 0$, so $y^{\prime} \leq 0$ and $y$ achieves its minimum at $x=1$. Now $y(1)<0$ would force $h\left(y^{\prime}(1)\right)>0$, implying that $y^{\prime}(1)>\beta \geq 0$ and so contradicting the minimality of $y(1)$. Thus all solutions of the extended problem are nonnegative and so solutions of the original problem.

In view of $\mathrm{H} 3$, the operator $N$ defined by

$$
(N y)(x)=-h\left(y^{\prime}(1)\right)+\mu \int_{x}^{1} \frac{1}{p(s)} \int_{0}^{s} p(t) q(t) g(t, y(t)) d t d s
$$

maps $\mathrm{C}_{0}^{1}[0,1]$ continuously into itself. As before, $N$ is also a compact map, and the fixed points of $\lambda N$ are solutions of $(7)_{\lambda}-(8)_{\lambda}$, and conversely. The nonlinear alternative again shows that $N$ has a fixed point in $\bar{U}$, which is the desired nonnegative solution of (1)-(2).

Remarks. Note that in this proof, unlike that of Theorem 1, we do not produce a bound on $p\left|y^{\prime}\right|$ for all solutions, but only for solutions satisfying $0 \leq y(x) \leq M_{0}$.

Theorem 2 sometimes gives stronger results than Theorem 1, which is, however, easier to use. For example, for the equation $y^{\prime \prime}+2 \mu y=0$ with $h(z) \equiv z$ Theorem 1 yields existence of a solution for $\mu<1 / 4$, while the condition of Theorem 2 is $\mu<0.339$, approximately.

\section{REFERENCES}

1. L. E. Bobisud, J. E. Calvert, and W. D. Royalty, Existence of biological populations stabilized by diffusion, Diff. Eqs. Dynamical Systems (to appear).

2. L. H. Erbe and H. Wang, On the existence of positive solutions of ordinary differential equations, Proc. Amer. Math. Soc. 120 (1994), 743-748. MR 94e:34025

3. A. Granas, R. B. Guenther, and J. W. Lee, Some general existence principles in the Carathéodory theory of nonlinear differential systems, J. Math. pures et appl. 70 (1991), 153-196. MR 92d:34041

4. D. O'Regan, Theory of singular boundary value problems, World Scientific, Singapore, 1994. MR 95g:34003

Department of Mathematics and Statistics, University of Idaho, Moscow, Idaho 83844-1103

E-mail address: bobisud@uidaho.edu

Department of Mathematics, University College Galway, Galway, Ireland

E-mail address: donal.oregan@ucg.ie 